(C) 1981. The Genetical Society of Great Britain

\title{
SPATIAL AND TEMPORAL VARIATION IN THE GENOTYPIC FREQUENCIES OF THE MUSSEL BRACHIDONTES ROSTRATUS*
}

\author{
D. J. COLGAN \\ Department of Genetics, University of Melbourne, Melbourne, Australia 3053 \\ Received 18.viii.8()
}

\begin{abstract}
SUMMARY
The results of three experiments on the population genetics of the Australian beaked mussel, Brachidontes rostratus are discussed in relation to the data which are avajlable on other mytilids. The experiments comprise studies of interpopulation genetic differentiation, microgeographic variation of leucineaminopeptidase genotypic frequencies in samples taken along a transect of one population and microgeographic and temporal variation in malate dehydrogenase genotypic frequencies. Natural selection is shown to be the most probable determinant of genotypic frequencies in $B$. rostratus. Selective coefficients are temporally and spatially variable. In common with other mussels, $B$. rostratus shows a deficiency of heterozygotes at the $L A P-1$ locus. Eight explanations are considered for the persistence of polymorphism at this locus. It is concluded that frequency-dependent selection and temporally and spatially variable fitnesses should be further investigated in relation to the $L A P-1$ polymorphism although it is not presently possible to explain the general finding of $L A P$ heterozygous deficits in mussels.
\end{abstract}

\section{INTRODUCTION}

THE answer to the question of the primacy of genetic drift or differential fitness in the determination of genotypic frequencies can only be ascertained by the investigation of large numbers of loci from diverse species. The general biology and ecology of mussels is well researched (Loosanoff and Davies, 1963; Mason, 1972; Bayne, 1976; Seed, 1976), and three features of their biology make them particularly attractive to population genetic investigation. The first is that it is possible to define populations of adults because such individuals are sessile-or nearly so (Harger, 1972). Secondly, mussels have a very large reproductive excess for the manifestation of any selective differentials which may exist. Thirdly, a wide variety of agents is known to cause mortality in mussels when they are available for study on rock platforms (Seed, 1976). Such agents may be associated with differential fitness.

The study of the population genetics of mussels has been largely restricted to the genus Mytilus in general and to M. edulis and M. californianus in particular (reviewed in Levinton and Koehn, 1976). The present paper reports experiments which have been undertaken to study the population genetics of a species of another genus, the beaked mussel Brachidontes rostratus. These experiments utilized the genetic variation in $B$. rostratus

* This paper is dedicated to Dr G. C. Hipwell, Dr E. Davis, Dr U. Christopher and the Staff of the Royal North Shore Hospital.

$\dagger$ resent Address: School of Biological Sciences, Botany-Macleay Building, University of Sydney, N.S.W., Australia 2006. 
which has been revealed by electrophoresis (Colgan, 1980), in an attempt to demonstrate the probability of the operation of natural selection on the loci of this species. The results of these experiments can also be compared to the results of similar experiments using Mytilus species to allow the identification of characteristics of their population genetics which are common to mussel species.

\section{MATERIALS AND METHODS}

Collection and storage methods, electrophoretic techniques, details of population localities and times of collection are given in Colgan (1980). The distribution is arbitrarily divided into three regions. The Mallacoota region contains the single outlying population, the Tasmanian region contains the Swansea and Bicheno populations and the Central Region comprises the remaining thirteen populations. Pairs of populations less than one kilometre apart were sampled at Koonya, Lorne and Skene's Creek Bay.

Measurement of length, height and thickness of shells was performed with Vernier callipers. The length is defined as the longest anterior-posterior axis of the shells; the height is the greatest dorsal-ventral axis and the thickness is the largest width of the conjoined valves.

\section{Results}

\section{(i) Inter-population and inter-region differences in genotypic frequencies}

The allele frequencies found in the Central Region and the observed and expected heterozygosities are given for all of the studied loci of $B$. rostratus in Colgan (1980). This section is concerned with variation in the genotypic frequencies of the local populations which are used to arrive at the composite regional frequencies. It is also concerned with differences between the frequencies of the various regions.

The polymorphic loci which have been scored in three or more populations are esterase-5 (Est-5), leucineaminopeptidase-1 and -2 (LAP-1, $L A P-2)$, malate dehydrogenase-1 and $-2(M D H-1, M D H-2), \alpha$-glycerophosphate dehydrogenase-2 $(\alpha-G P D H-2)$, xanthine oxidase $(X O)$ and tetrazolium oxidase-4 (TO-4). The slow allele at the $X O$ and TO-4 loci is too rare for meaningful tests of geographic heterogeneity within the Central Region. The $G$-test of homogeneity (Sokal and Rohlf, 1969) of genotypic frequencies is marginally significant at the Est-5 locus with a small sample size of 42 . The result of a $G$-test is near significance for the $\alpha-G P D H-2$ genotypic frequencies of the Central Region $(0 \cdot 1>P>0 \cdot 05)$. There is significant geographic variation in the $M D H-1$ frequencies in the Central Region $(P<0.005)$. In particular, the genotypic distributions of the Point Lonsdale and Barwon Heads populations are different $(P<0 \cdot 05)$. The allelic frequencies are similar in these two populations but the combination of alleles differs to the extent that the Point Lonsdale population is out of Hardy-Weinberg equilibrium whilst the Barwon Heads population conforms to expectation. A $G$-test does not reveal whatever inter-population variation exists at the $M D H-2$ locus $(0 \cdot 75>P>0 \cdot 5)$.

The genotypic frequencies of the $L A P-1$ locus are shown in table 1 with an indication of the probability that a population is in Hardy-Weinberg 
TABLE 1

Leucineaminopeptidase-1 genotypic frequencies in various localities

$\begin{array}{lcccccccccc}\text { Site } & \mathrm{FF}^{\dagger} & \text { FM } & \text { MM } & \text { FS } & \text { MS } & \text { SS } & \text { f } & \text { m } & \text { N } & \text { Pr } \\ \text { Mallacoota } & 0.14 & 0.27 & 0.57 & 0.03 & & & 0.28 & 0.70 & 37 & >0.5 \\ \text { Bicheno } & 0.54 & 0.13 & 0.21 & & 0.04 & 0.08 & 0.60 & 0.29 & 24 & <0.005 \\ \text { Swansea } & 0.04 & 0.06 & 0.45 & 0.02 & 0.08 & 0.33^{*} & 0.08 & 0.54 & 49 & <0.005 \\ \text { San Remo } & 0.08 & 0.25 & 0.61 & 0.03 & 0.03 & & 0.22 & 0.75 & 36 & >0.5 \\ \text { Koonya } & 0.17 & 0.17 & 0.53 & 0.03 & 0.11 & & 0.26 & 0.67 & 36 & >0.05 \\ \text { Portsea } & 0.03 & 0.20 & 0.68 & & 0.07 & 0.02 & 0.13 & 0.81 & 59 & >0.9 \\ \text { Point Lonsdale } & 0.13 & 0.13 & 0.60 & 0.01 & 0.11 & 0.03 & 0.20 & 0.72 & 112 & <0.005 \\ \text { Barwon Heads } & 0.08 & 0.20 & 0.60 & 0.04 & 0.08 & & 0.20 & 0.74 & 25 & >0.5 \\ \text { Torquay } & 0.18 & 0.30 & 0.44 & 0.03 & 0.05 & & 0.34 & 0.62 & 61 & >0.1 \\ \text { Point Roadknight } & 0.05 & 0.29 & 0.53 & 0.08 & 0.03 & 0.03 & 0.24 & 0.68 & 38 & >0.75 \\ \text { Lorne } & 0.25 & 0.28 & 0.58 & 0.04 & 0.06 & & 0.41 & 0.55 & 53 & >0.05 \\ \text { Artillery Rocks } & 0.26 & 0.29 & 0.39 & 0.03 & & & 0.44 & 0.53 & 31 & >0.1 \\ \text { Kennett River } & 0.17 & 0.22 & 0.56 & 0.02 & 0.02 & & 0.30 & 0.68 & 82 & <0.005 \\ \text { Skene's Creek } & 0.17 & 0.29 & 0.52 & 0.02 & & & 0.32 & 0.67 & 42 & >0.1 \\ \quad \text { Bay E. } & & & & & & & & & & \\ \text { Skene's Creek } & 0.07 & 0.23 & 0.53 & 0.05 & 0.10 & 0.02 & 0.21 & 0.70 & 60 & >0.25 \\ \quad \text { Bay W. } & & & & & & & & & & \\ \quad \text { Total } & 0.06 & 0.21 & 0.52 & 0.03 & 0.06 & 0.03 & 0.26 & 0.66 & 745 & <0.001\end{array}$

$\dagger \mathrm{F}, \mathrm{M}$ and $\mathrm{S}$ are allele designations, $\mathrm{f}, \mathrm{m}$ and $\mathrm{s}$ allele frequencies, $\mathrm{N}$ the number of individuals examined and $\operatorname{Pr}$ the probability of Hardy-Weinberg equilibrium.

* Includes one slow-very slow heterozygote.

equilibrium at the locus. $G$-tests show that there is a significant interpopulation variation in $L A P-1$ frequencies in both the Tasmanian $(P \ll$ $0.005)$ and Central Regions $(P<0.025)$.

Genetic distances between populations have been calculated from Nei's (1975) formula for the "standard genetic distance". The sampling variance of this formula can be calculated only when the populations are in HardyWeinberg equilibrium (Nei and Roychoudhury, 1974). The genetic distance (based on 10 loci) between the Point Lonsdale and Barwon Heads populations is $0 \cdot 05$. The distance between Koonya and Skene's Creek Bay (4 loci) is 0.043 .

Five loci have been scored in more than one region. These are the $L A P-1$ and $L A P-2, \alpha-G P D H-2$, and TO-2 and TO-4 loci. There is significant inter-regional heterogeneity in genotypic frequencies in both LAP-1 $(P \ll 0.005)$ and $\alpha G P D H-2(P \ll 0.005)$. The $\alpha G P D H F$ and $S$ alleles have not been found in the 38 individuals scored from Tasmania. Hook's (1977) tables give a 95 per cent confidence limit for the frequency of an undetected variant as 4 per cent for this number of individuals, each with two alleles. A $G$-test of heterogeneity at the LAP-2 locus is nearly significant $(0 \cdot 1>P>0 \cdot 05)$. The TO-4 locus is nearly monomorphic. The slow allele at this locus is absent from the Mallacoota and Tasmanian samples. The 95 per cent confidence limit for the frequency of an undetected variant is 4 per cent for Mallacoota, 3 per cent for Tasmania and 2 per cent for the pooled data.

There are two loci with marked deviations from Hardy-Weinberg equilibrium in one or several regions. All three $L A P-1$ heterozygotes are significantly rarer than expected in Tasmania and the $F M$ heterozygote is significantly rare in the Central Region. There is a non-significant excess of 
the FS genotype at Mallacoota but all other heterozygous classes of the LAP-1 locus show (non-significant) deficits. The single population at Mallacoota is significantly out of Hardy-Weinberg equilibrium for the $\alpha-G P D H-2$ locus. The Central Region is also out of Hardy-Weinberg for this locus.

The genetic distance between Mallacoota and Tasmanian regions is 0.081 ; between Mallacoota and the Central Region 0.051 ; and between Tasmania and the Central Region 0.019.

\section{(ii) LAP-1 genotypic frequencies in a transect at Point Lonsdale}

Microgeographic variation in genotypic frequencies was investigated in an analysis of $419 L A P-1$ genotypes in twenty samples from a sea-to-shore transect of the Point Lonsdale population of B. rostratus. Sample height above or below a reference, and distance shoreward from the origin of the transect were recorded. The value of the variable $k=\left(\mathrm{Het}_{0}-\mathrm{Het}_{e}\right) / \mathrm{Het}_{e}$ was obtained for each sample point. This variable is a measure of the deviation of observed heterozygosity from Hardy-Weinberg expectation (Selander, 1970). Only one sample is out of Hardy-Weinberg equilibrium according to the $\chi^{2}$ test. However a sign test of the values of $k$ is significant at the 0.001 level. Only the small sample from point 18 has an excess of heterozygotes.

There is a significant difference in the $L A P-1$ genotypic frequencies found in the geographic survey and in the present experiment. The two collections were made 6 months apart on the opposite sides of a large rock at the site. The result of a $\chi^{2}$ test of heterogeneity comparing genotypic frequencies in the geographic survey and the pooled data from the four highest points of the transect is significant at the $0 \cdot 001$ level so that the heterogeneity is not simply an artefact of the method of collection used for the geographic survey of sampling from the top of the local range.

A $G$-test of the association of genotype and site along the transect is not significant $(0 \cdot 5>P>0 \cdot 25)$. There is no correlation of genotypic and allelic frequencies with shoreward distance or sample point height. But there is evidence of microgeographic variation in genotypic frequencies along the transect to be found in the significant correlation of site location and $k$, the coefficient of heterozygote deficit. The correlation is significant at the 0.05 level and indicates an increase of the deficit of heterozygotes in the seaward direction.

The length, breadth and thickness and the hinge-teeth phenotype of the shells were recorded for each mussel taken from the transect. The average lengths of mussels of a given genotype have been compared using a $t$-test. The length of $F F$ individuals is generally less than that of other genotypes (significantly so for $F M, M M$ and $M S$ ) but there are no major differences between other genotypes. No significant effect of genotypes is found in a two-way analysis of variance of the effect of site and genotype on shell length. The effect of site on length is very significant $(P<0 \cdot 001)$ and there is a significant interaction between site and genotype $(P<0 \cdot 02)$. There is a significant result from the $G$-test of association between genotype and (arbitrarily defined) size-classes. The frequency of the $F$ allele in the 5 classes $(9-13 \mathrm{~mm}, 13-17 \mathrm{~mm}, 17-21 \mathrm{~mm}, 21-25 \mathrm{~mm},>25 \mathrm{~mm})$ is 0.26 , $0 \cdot 28,0 \cdot 26,0 \cdot 24$, and $0 \cdot 25$. The $13-17 \mathrm{~mm}$ class is the only one to return a 
near significant result of the $G$-test of association of site and genotype $(P=0 \cdot 1)$.

A linear regression of mussel length on site at Point Lonsdale is significant but the large residual sum of squares argues that the relation of length and shoreward distance is not simply linear. This is confirmed by the analysis of the lengths of 620 mussels sampled from various points along a transect at San Remo. Linear, quadratic and cubic contrasts were made of the points in this collection. The linear term is very significant $(P=0 \cdot 001)$, the quadratic is not significant and the cubic is marginally significant $(0.05>P>0.04)$. The remnant sum of squares is significant at the 0.001 level. The pattern is similar for tests of shell breadth and thickness.

The number of age-classes in the populations at Point Lonsdale and San Remo has been estimated by applying the analysis of Bhattacharya (1967) to the length frequency data. The two identifiable classes at Point Lonsdale are equivalent in size range to two of the four classes found at San Remo. In view of the interaction of site and length in the two populations, criticism of graphical methods of resolving mixtures of distributions (Macdonald and Pitcher, 1979) and the lack of success in previous attempts to age mussels from length data (Seed, 1976), it seems unwise to ascribe a significance to the size classes which have been identified.

\section{(iii) The temporal stability of malate dehydrogenase genotypic frequencies}

Microgeographical variation and temporal stability of malate dehydrogenase genotypic frequencies were investigated in 24 samples in two collections taken a year apart from a population on a $3 \times 2 \mathrm{~m}$ rock at Barwon Heads. Full details of methods and results (for the 912 genotyped individuals) are given in Colgan (1980). The two main points to emerge are that there is significant microgeographical variation in the $M D H-1$ genotypic frequencies in both collections and that there are changes in genotypic frequencies between the collections. The frequency changes were not uniform over the rock. A difference discovered in the genotypic frequencies of the eastern and western halves of the rock in the second collection was not found in the first collection. It is not possible to prove that selective mortality is responsible for changes in genotypic frequency because it was not possible to identify, by length frequency analysis, a cohort which was certainly represented in both collections (Colgan, 1980).

\section{Discussion}

\section{(i) Hardy-Weinberg equilibrium}

At least three of the enzyme loci examined in $B$. rostratus are out of Hardy-Weinberg equilibrium in one or more populations. There are deficits of heterozygotes at the LAP-1 and $\alpha-G P D H-2$ loci and an excess at the Point Lonsdale population of the $F S$ genotype of the $M D H-1$ locus. Heterozygous deficiency is known for loci in a number of marine organisms (Gaines, Caldwell and Vivas, 1974; Koehn, Turano and Mitton, 1973). It is noteworthy that a deficit of heterozygotes has been found at an $L A P$ locus in every mussel thus far studied. There are deficits in M. edulis (Mitton and Koehn, 1973; Levinton and Fundiller, 1975; Koehn, Turano and Mitton, 
1973; Lassen and Turano, 1978), for M. californianus (Tracey, Bellet and Gravern, 1975) and M. galloprovincialis (Ahmad and Beardmore, 1976). The $L A P-1$ data from $B$. rostratus may be adduced with these reports to the contention that $L A P$ heterozygote deficit is the rule in mussels. The $M D H$ data may be cited as showing that not every mussel locus has such a deficit. There is evidence from M. edulis of a decrease in the magnitude of the deficit in larger size classes (Levinton and Fundiller, 1975; Levinton and Koehn, 1976) but even the largest size class of the mussels from Point Lonsdale is significantly out of Hardy-Weinberg equilibrium for the LAP-1 locus.

\section{(ii) Geographic variation in genotypic frequencies}

The pattern of population variation in genotypic frequencies is different in $M$. edulis and B. rostratus. Major frequency changes in B. rostratus occur in short distances but are not accompanied by the regional invariance which is found in $M$. edulis. The frequency of the $L A P^{94}$ allele is uniform in $M$. edulis from Virginia to Cape Cod but declines abruptly to the frequency found throughout the Gulf of Maine (Koehn, Milkman and Mitton, 1976). The six British populations surveyed by Ahmad, Skibinski and Beardmore (1977) show no variation in $L A P$ gene frequencies. There was no variation in these populations in the $A P-1, E s t-D, P G M$ or $P H I$ frequencies. Koehn et al. (1976) report a clinal change in allele frequencies of the GPI locus in North American populations. No evidence of a cline has been found in any $B$. rostratus locus.

Geographic variation in $M$. californianus is similar to that in B. rostratus. The $L A P-1$ locus of $M$. californianus exhibits as much variation in allelic frequency on the small island of Tatoosh, near Vancouver, as it does in its range of $24^{\circ}$ of latitude along the west coast of North America (Levinton and Suchanek, 1978). This pattern is repeated in GPI frequencies.

The synthesis of the variation of allele frequencies into the genetic distance between populations allows a (non-rigorous) test of the action of selection in the maintenance of genetic differentiation. "If interpopulation gene flow were important in genetic similarity we would predict that interlocality differences over the largest scale of geography would be the greatest" (Levinton and Koehn, 1976). This is not the case in B. rostratus where the distance between a pair of neighbouring populations in the Central Region is larger than the distance between locales near the extremes of the region and where inter-regional differences are of the same order as interpopulation differences. The interpretation that selective pressures are of predominant importance in interpopulation differentiation is stated by Levinton and Koehn (1976) on the basis of similar results using Hedrick's coefficient of genetic similarity from data from $M$. edulis and $M$. californianus.

The finding of microgeographic variation in $M D H$ frequencies is not unexpected in view of the unqualified reports of variation in genotypic frequencies of near sites (Levinton, 1973; Levinton and Koehn, 1976). Koehn, Turano and Mitton (1973) studied the TO locus of Guekensia demissa and found an increased deficit of heterozygotes at the higher sample point of two. Similar results were obtained for the deficits of heterozygotes in samples from high and low points in the range of $M$. californianus (Levinton and Fundiller, 1975). 
The results of the transect experiment at Point Lonsdale are directly comparable to those of the study of two transects of one population of $M$. edulis by Balegot (1971). One transect showed a positive correlation between the deficiency of $L A P$ heterozygotes and distance from the shore. There was no correlation along the second transect. The correlation is negative in the Point Lonsdale population.

\section{(iii) Temporal changes in genotypic frequency}

There have been a number of temporal studies of isoenzyme frequencies in molluscs. There is significant age-dependent variation in gene frequencies at the $L A P$ locus of $G$. demissa (Schopf, Ohman and Bleiweiss, 1975). Buroker (1979) has shown heterozygous advantage at the muscle protein-1 locus of the Japanese oyster, Crassostrea gigas in longitudinal studies of three age-classes sampled from the bottom of the species range. The study by Singh and Zouros (1978) of 4 loci of the American oyster, $C$. virginica, found that laboratory culture for one year was associated with departures from Hardy-Weinberg equilibrium at the loci. A study of the $L A P$ genotype of newly settled individuals in 3 successive cohorts of $M$. edulis on Tatoosh Island has found no temporal variation (Levinton and Koehn, 1976). The study of $M D H$ genotypic frequencies in the Barwon Heads population has revealed significant changes. It has also confirmed that the pattern of frequency changes need not be random on the rock platform.

(iv) Explanation of the genotypic frequency patterns of B. rostratus

Four hypotheses may be postulated, singly or in combination, as causes of the genotypic frequency patterns. It is probable that all the factors invoked have a role in the evolution of mussel populations but the following discussion is aimed at establishing the primary determinants of genotypic frequency. The hypotheses are:

(1) Patterns of population recruitment are changing over time.

(2) Settlement preferences among the genotypes are associated with point-specific growth parameters.

(3) There is an interaction of mussel genotype and point-specific growth parameters.

(4) Temporally and spatially variable natural selection is operative on the studied loci or on a locus in linkage disequilibrium.

Genetic differentiation between local populations might be explained, under the first hypothesis, by alternative causes. Genotypic frequencies in a common larval pool may be changing over time so that populations with a diverse age structure have different compositions. Secondly, there may be differences in the larval pools from which the settling mussels are recruited to the adult population. It is necessary in either case to explain the origin of the temporal or spatial variation in the composition of larval pools.

It seems unlikely that this hypothesis can explain the microgeographic variation in $L A P-1$ and $M D H-1$ genotypic frequencies. The correlation of heterozygote deficit and sample point on the Point Lonsdale transect would not be expected under this hypothesis. The length-frequency analysis of the second collection at Barwon Heads has shown that this collection may 
represent one or two age classes (Colgan, 1980). If there are two classes then the homogeneity of the frequency of $M D H-1$ genotypes over the length classes shows that the adult population composition is not affected by such differences as do exist in the larval pools of the cohorts. If only one cohort is represented the hypothesis cannot be used as an explanation of the microgeographic variation in this collection.

The second hypothesis may be used to explain the apparent deficits of heterozygotes in geographic surveys as an artefact of the method of sample collection. These samples were taken from the top of the species range in a locality. If heterozygotes have a preference for lower areas of the range the deficit in the samples may not represent the genotypic composition of the overall population. The increase of heterozygous deficit in the seaward direction is against this. A deficit of heterozygotes at all levels of the range in another transect or in a geographic survey made at a second level of the range is necessary for the conclusion that the deficit is not artefactual. Balegot's (1971) study shows that two transects of the same population might well lead to different conclusions about heterozygous deficit.

The relation between $L A P-1$ genotype and shell length might be explained as the effect of site dependent (genotype independent) growth differences after the induction of microgeographic heterogeneity by settlement preferences. It would be expected that the confusion of size-classes which would result from such growth would lead to inhomogeneity along the transect in a single class unless there is genotypic uniformity between year classes. No such inhomogeneity has been found although only one pair of adjacent size classes $(13-17 \mathrm{~mm}, 17-21 \mathrm{~mm})$ differs significantly in genotypic composition.

There is a positive correlation of length and seaward distance in the mussels at Point Lonsdale (Colgan, 1980). The heterozygote deficit is larger nearer the sea. But there is a tendency for $F M$ heterozygotes to be larger than $F F$ homozygotes. These three facts imply that there must be interactions between genotype and site in the determination of growth. The third hypothesis, that there is an interaction of site and genotype in the determination of growth, is, then, a necessary adjunct to the second hypothesis of changeable genotype-dependent settlement patterns.

The final testing of the hypothesis of changing non-random settlement must await the investigation of the dispersal of a cohort of very young individuals over the surface of a rock platform. At the moment it can only be said that the apparent overall heterozygote deficit is against the hypothesis.

The third hypothesis cannot alone be the explanation of observed genotypic frequency patterns because it does not explain heterozygous deficits or interpopulation variation at a locus. The hypothesis must be considered in relation to one or more of the remaining three.

It can be hypothesized that the selective coefficient of any genotype is determined by local conditions and may be temporally variable. But it must be shown, before this hypothesis is accepted, how it can provide for the apparent maintenance of the $L A P-1$ polymorphism. All 3 common alleles of the $L A P-1$ system are found in all three regions of the distribution of $B$. rostratus. It is unlikely that migration between the regions is enough to rectify the loss of an allele from a region which might be caused by selective elimination due to the disadvantage of heterozygotes or homozygotes. The finding of an $L A P$ polymorphism which has heterozygous deficit in all 
studied mussels is also an argument in favour of the maintenance of the polymorphisms.

It is known that loci with three or more alleles may be maintained in stable equilibrium even when the fitness of one heterozygote is not superior to the fitness of any homozygote (Crow and Kimura, 1970). For stability, however, population mean fitness at equilibrium must exceed the fitness of any homozygote. This is not possible if all the heterozygotes are less fit than all homozygotes. All heterozygotes are apparently less fit than all homozygotes in the $L A P-1$ system of $B$. rostratus. But the appearance of heterozygous disadvantage in Hardy-Weinberg analysis of a single sample may be artefactual: only one of the homozygotes need be favoured over the heterozygote to give this appearance (Lewontin and Cockerham, 1959). The appearance of three heterozygote deficits (out of three classes) necessitates, however, that one of the homozygotes must be fitter than each other genotype. This means that the polymorphism should be unstable. An explanation other than constant relative fitnesses must be sought for the persistence of the $L A P$ polymorphism.

(v) The maintenance of polymorphisms with an apparent deficit of heterozygotes

Null alleles alter the expected frequencies of electrophoreticallydetermined phenotypes. Expectations for phenotypic frequency can be found if it is assumed that the null homozygote is lethal (no such individuals were observed for the $L A P-1$ locus of $B$. rostratus). The revised allelic frequencies can be abstracted from the observed phenotypic frequencies and applied to the expectations. A null allele which could be responsible for the heterozygote deficit in the pooled Point Lonsdale samples would have an appreciable frequency of $0 \cdot 15$. The $\chi^{2}$ value of the test of the observed phenotypic frequency to revised expectation is $2 \cdot 66$. Despite this, it does not seem likely that null alleles can be a general explanation of heterozygous deficit in mussels (Kartavtsev, 1978).

A simple failure to distinguish between phenotypes might explain an isolated report of heterozygote deficit but it would not explain the widespread occurrence of the deficit at mytilid $L A P$ loci. It is possible that a specialized system of the control of gene expression in Mytilidae may be responsible for the deficits. Misscoring may result if an allele is dominant but has variable penetrance and expressivity. The hybrid of two fish species exhibits, for instance, an amylase phenotype which has a reduced amount of the isoenzyme of one species (Herrerra, 1979). No variation of this type has been noted in $B$. rostratus but hypotheses of genetic control cannot be rejected until breeding studies have been performed on this or other mussels.

The Wahlund effect has been proposed as the cause of heterozygous deficits in some marine species. Koehn et al. (1976) have shown, however, that the effect is too small to explain $L A P$ heterozygous deficits in $M$. edulis. The effect is also too small to explain $\alpha-G P D H-2$ or $L A P-1$ deficits in $B$. rostratus. The estimate of allelic frequency variance which was used to make corrections to the expected genotypic frequencies was the sample variance of allele frequencies in the Central Region. 
The hypothesis that the $L A P-1$ locus is maintained by a heterozygous advantage in fertility which compensates for a disadvantage in viability seems implausible because of the mode of reproduction in mussels. Advantage could only derive from the numbers of gametes spawned. The hypothesis was, however, applied to the Point Lonsdale $L A P-1$ transect data to ascertain what degree of fertility advantage would be necessary to maintain the population in the present genotypic composition which is assumed to represent a stable equilibrium. The $F M$ heterozygote would have to be $15 \cdot 2$ times as fertile as the $F F$ and $M M$ homozygotes. This figure is unlikely to be achieved.

The polymorphism could be maintained by frequency dependent selection in favour of rare genotypes. No test of this possibility could be made using the available data on $B$. rostratus. A test of this factor would require data from two stages of a life-cycle or two generations.

Inbreeding reduces the expected frequency of heterozygotes (Crow and Kimura, 1970). The coefficient of inbreeding is equal to $-k$. The value of the coefficient for the pooled transect data on the $L A P-1$ polymorphism is 0.258 which is equivalent to a full-sib mating. This level of inbreeding is much too high for open populations of dioecious species. The sexual states of $B$. rostratus are unknown. But even if this mussel is self-fertilizing it is not possible that inbreeding is a general explanation of heterozygote deficit because the vast majority of $M$. edulis are unisexual (Seed, 1976).

Polymorphisms with variable genotypic fitnesses may be maintained if the geometric mean fitness of the heterozygote is greater than that of either homozygote (Gillespie, 1973). If the fitness of the two homozygotes are $(1+r)$ and $(1+s)$ in one generation and $(1+s)$ and $(1+r)$ in the next, it is necessary for maintenance, if the heterozygous fitness is always 1 , that $r+s+r s<0$. The condition that the sample shows a deficit of heterozygotes is that $r+s+r s>0$ (Lewontin and Cockerham, 1959). Both conditions cannot be satisfied.

Gillespie's condition could be satisfied in the long term if the appearance of heterozygous deficit is rare and if selection against the homozygotes is large when there is no deficit in a generation. But there is FM LAP-1 heterozygous deficit in all fourteen surveyed populations. Hence it is unlikely that the $L A P-1$ polymorphism is stable because it is maintained by the temporal variation of selective coefficients.

The polymorphism might be persistent because of the temporal and geographic variation of selective coefficients. The appearance of heterozygous disadvantage can be induced by selection in favour of either homozygote; but it is not possible to identify the favoured genotype by examination of one sample (Lewontin and Cockerham, 1959). The various LAP-1 homozygotes may each be favoured in different areas (and at different times) with the result that the actual overall selective coefficients are quite similar. The puzzle remains, however, as to why the actual selective coefficients should be such that the relation $r+s+r s>0$ is generally satisfied in $B$. rostratus populations.

In conclusion, it can be stated that only the Wahlund effect and inbreeding can be rejected as causes of the apparent heterozygous disadvantage. Sex linkage, null alleles, misscoring and heterozygous advantage in fertility are unlikely to be of importance but will have to be excluded by breeding studies. Frequency dependent selection and temporal and geographic vari- 
ation in fitness seem at present to be the most likely explanations of the maintenance of polymorphisms with an apparent heterozygous deficit. Since natural selection is the most likely hypothesis in the explanation of genotypic frequencies in $B$. rostratus, the investigation of these two factors assumes paramount importance in the prospect of subsequent work on this species.

Acknowledgements.-I would like to thank Dr M. J. Whitten for reading the drafts of the manuscript and for his supervision of the work on which the manuscript is based. I also thank Dr D.F. Gartside for his advice during the progress of the work. I wish to thank Ms L. Duff, Ms A. Graham and Mr E. Page for help in the production of the manuscript.

\section{REFERENCES}

Ahmad, M., And Beardmore, J. A. 1976. Genetic evidence that the "Padstow Mussel" is Mytilus galloprovincialis. Mar. Biol., 35, 139-147.

AHMAD, M., SKIBINSKI, D. O. F., AND BEARDMORE, J. A. 1977. An estimate of the amount of genetic variation in the common mussel, Mytilus edulis. Biochem. Genet., 15, 833-846.

BALEGOT, P. P. 1971. Microgeographical variation at two biochemical loci in the blue mussel, Mytilus edulis. M.A. Thesis, State University of New York, Stony Brook.

BAYNE, B. L. 1976. The biology of mussel larvae. In IBP 10, Marine Mussels, ed. B. L. Bayne, pp. 81-120. Cambridge University Press, Cambridge.

BHATTACHARYA, C. G. 1967. A simple method of resolution of a distribution into Gaussian components. Biometrics, 23, 115-135.

BUROKER, N. E. 1979. Overdominance of a muscle protein (Mp-1) locus in the Japanese oyster, Crassostrea gigas (Ostreidae). J. Fish. Res. Board Can., 36, 313-318.

COLGAN, D. J. 1980. Theoretical and practical studies on genetic fitness. Ph.D Thesis, University of Melbourne, Melbourne.

CROW, J. F., AND KIMURA, M. 1970. An Introduction to Population Genetics Theory. Harper and Row, New York

GAINES, M. S., CALDWELL, J., AND VIVAS, A. M. 1974. Genetic variation in the mangrove periwinkle, Littorina angulifera. Mar. Biol., 27, 327-332.

GiLlesPiE, J. 1973. Polymorphism in random environments. J. Theor. Pop. Biol., 4, 193-195.

HARGER, J. R. E. 1972. Competitive co-existence among intertidal invertebrates. Am. Scient., $60,600-607$.

HERRERRA, R. J. 1979. Preferential gene expression of an amylase allele in interspecific hybrids of Xiphophorus (Pisces; Poeciliidae). Biochem. Genet., 17, 223-227.

HOOK, E. B. 1977. Exclusion of chromosomal mosaicism: tables of 90 per cent, 95 per cent and 99 per cent confidence limits and comments on use. Am. J. Hum. Genet., 29, 94-97.

KARTAVTSEV, Y. F. 1978. Genetic variability of a bivalve mollusk, the mussel Crenomytilus grayanus. Soviet Genetics, 14, 186-190.

KOEHN, R. K., MILKMAN, R., AND MITTON, J. B. 1976. Population genetics of marine pelecypods IV. Evolution, 30, 2-32.

KOEHN, R. K., TURANO, F. J., AND MITTON, J. B. 1973. Population genetics of marine pelecypods II. Evolution, 27, 100-105.

LASERON, C. F. 1956. New South Wales mussels. Aust. Zool., 12, 263-283.

LASSEN, H. H., AND TURANO, F. J. 1978. Clinal variation and heterozygote deficit at the leucineaminopeptidase locus of Mytilus edulis. Mar. Biol., 49, 245-254.

LEVINTON, J. S. 1973. Genetic variation in a gradient of environmental variability: marine bivalvia. Science, 180, 75-76.

LEVINTON, J. S., AND FUNDILLER, D. 1975. An ecological and physiological approach to the study of biochemical polymorphisms. In Proc. IX. Eur. Mar. Biol. Symp., ed. H. Barnes, pp.165-176. Aberdeen University Press, Aberdeen.

LEVINTON, J. S., AND KOEHN, R. K. 1976. Population genetics of mussels. In IBP 10, Marine Mussels, ed. B. L. Bayne, pp. 357-384. Cambridge University Press, Cambridge.

LEVINTON, J. S., AND SUCHANEK, T. H. 1978. Geographic variation, niche breadth and genetic differentiation at different geographic scales in the mussels Mytilus edulis and Mytilus californianus. Mar. Biol., 49, 363-376. 
LEWONTIN, R. C., AND COCKERHAM, C. C. 1959. The goodness-of-fit test for detecting natural selection in random mating populations. Evolution, 13, 561-564.

LOOSANOFF, V. L., AND DAVIES, H. C. 1963. Rearing of bivalve molluscs. Adv. Mar. Biol., 1, 1-136.

MACDONALD, P. D. M., AND PITCHER, T. J. 1979. Age groups from size-frequency data: a versatile and efficient method of analyzing distribution mixtures. J. Fish. Res. Board Can., 36, 987-1001.

MASON, J. 1972. The cultivation of the European mussel, Mytilus edulis. Ann. Rev. Ocean. Mar. Biol, 10, 437-460.

MITTON, J. B., AND KOEHN, R. K. 1973. Population genetics of marine pelecypods III. Genetics, 73, 487-496.

NEI, M. 1975. Molecular Population Genetics and Evolution. Elsevier/North Holland, Amsterdam.

NEI, M., AND ROYCHOUDHURY, A.K. 1974. Sampling variances of heterozygosity and genetic distance. Genetics, 76, 379-390.

SCHOPF, T. J. M., OHMAN, M. D., AND BLEIWEISS, B. 1975. Significant age-dependent and locality-dependent changes occur in gene frequencies in the ribbed mussel, Modiolus demissus from a single salt marsh. Biol. Bull., 149, 446.

SEED, R. 1976. Ecology. In IBP 10, Marine Mussels, ed. B. J. Bayne, pp. 13-66. Cambridge University Press, Cambridge.

SELANDER, R. K. 1970. Behaviour and genetic variation in natural populations. Am. Zool., 10, 53-66.

SINGH, S. M., AND ZOUROS, E. 1978. Genetic variation associated with growth rate in the American oyster (Crassostrea virginica). Evolution, 32, 342-353.

SOKAL, R. R., AND ROHLF, F. J. 1969. Biometry. W. H. Freeman, San Francisco.

TRACEY, M. L., BELLETT, N. F., AND GRA VERN, C. D. 1975. Excess allozyme homozygosity and breeding population structure in the mussel, Mytilus californianus. Mar. Biol., 32, 303-311. 